\title{
Solutions of Impulsive Diffusion and Von-Foerster-Makendrick Models Using the B-Transform*
}

\author{
Benjamin Oyediran Oyelami, Samson Olatunji Ale \\ National Mathematical Centre, Abuja, Nigeria \\ Email: boyelami2000@yahoo.com, aleola@ymail.com
}

Received October 2, 2013; revised November 2, 2013; accepted November 9, 2013

Copyright (C) 2013 Benjamin Oyediran Oyelami, Samson Olatunji Ale. This is an open access article distributed under the Creative Commons Attribution License, which permits unrestricted use, distribution, and reproduction in any medium, provided the original work is properly cited.

\begin{abstract}
In this paper we explore the possibility of using the scientific computing method to obtain the inverse B-Transform of Oyelami and Ale [1]. Using some suitable conditions and the symbolic programming method in Maple 15 we obtained the asymptotic expansion for the inverse B-transform then used the residue theorem to obtain solutions of Impulsive Diffusion and Von-Foerster-Makendrick models. The results obtained suggest that drugs that are needed for prophylactic or chemotherapeutic purposing the concentration must not be allowed to oscillate about the steady state. Drugs that are to be used for immunization should not oscillate at steady state in order to have long residue effect in the blood. From Von-Foerster-Makendrick model, we obtained the conditions for population of the specie to attain super saturation level through the "dying effect" phenomenon ([2-4]). We used this phenomenon to establish that the environment cannot accommodate the population of the specie anymore which mean that a catastrophic stage $t^{*}$ is reached that only the fittest can survive beyond this regime $\left(\right.$ i.e. $\left.t>t^{*}\right)$ and that there would be sharp competition for food, shelter and waste disposal etc.
\end{abstract}

Keywords: B-Transform; Impulsive Diffusion; Von-Foerster-Makendrick Models; Residue Theorem; Maple Symbolic Programme and Asymptotic Expansion

\section{Introduction}

Impulsive differential equations (IDEs) describe processes which quickly change their states for short moment of times when compare to the total evolution time for the systems. The impulses may happen at fixed or non-fixed moments and the behavior of state variables describing the processes may show some "jumps", "shocks" attributes etc. This kind of impulsive behavior makes the IDEs not easily accessible to most existing concepts and theories in differential equations, ecology, biomathematics, engineering and control systems ([2-9]). Moreover, impulsive moments not only depend on some impulsive sets but also on the dynamics of the systems, this special feature gives rich perspective for investigation for many real life processes $([2,3,8,10])$.

B-transform is an operational calculus method developed by Oyelami and Ale almost a decade ago for

\footnotetext{
"Dedicated to: Professor V. Lakshimikantham who has contributed extensively to the development of Differential Equations, Impulsive Differential Equations and Nonlinear Analysis.
}

finding closed solution forms for fixed moment impulsive systems ([1]). Most known existing transform methods are not known to be applicable to the (IDEs). It's interesting to say that the evolution times describing (IDEs) are intermittently interrupted by small perturbations (impulses) at certain fixed or non-fixed moments. This peculiarity makes the solutions of IDEs to exhibit some strange behaviors. Therefore any transform method for finding solution of IDEs must take into consideration the impulsive effect of the systems. The B-transform was developed to take into consideration the impulsive effect of the system to provide a method for finding solutions for the fixed moments IDEs ([1,11-13]).

B-transform has been successfully applied to solve a variety of problems in Biomedicine, Physics, engineering, control theory etc (see $[1,7,12])$. One major problem that one encounters just like most known transform methods ([1]) is how to obtain in the inverse transform for some particular problems ([14]). In this paper we explore the possibility of introducing scientific computing method to obtain the inverse B-Transform. We would make use of 
some suitable conditions and the symbolic programming method in Maple 15 to obtain the asymptotic expansion of the inverse B-transform, then followed by the use of residue theorem to obtain solutions to Impulsive Diffusion and Von-Foerster-Makendrick models.

We note that, the Impulsive Diffusion model is very useful for modelling the diffusion of drug across the cells and tissues in the body and the attendant osmo-regulation in the apparent volume and also modelling of environmental degradation problems $([5,15])$. More recently, stochastic diffusion models are used for analyzing behavior of financial markets. The entropy trap threatens the existence of man as a result of continuous emission of carbon dioxide into the air and water. The earth is getting more saturated carbon dioxide than the oxygen that plants absorb and release. The sea water is fast becoming acidic because of dissolution of carbon dioxide in the water and hence the marine lives are also threatened. The diffusion model considered here or its modifications have potential applications for studying diffusion of carbon dioxide into the atmosphere and the sea and the long time chaotic effect can be studied from impulsive perspective.

The theory of taxism, loosely speaking is the tendency of an organism to move forward or be away from some stimuli. There are many taxes, positive and negative which influence presumably direct organisms in favorable physical environments ([15]). Most notable or perhaps widely studied taxes are the chemo taxi that is response for organisms to be sensitive to chemical changes. If the response is measured in term of concentration of such chemicals, the impulsive diffusion model will give a clue to study chemo taxis of the expected organisms. More research works are expected to be on taxes in cellular ecology, that is, molecular and cellular biology. The study of bacterial and viral chemotaxes from impulsive point of view will offer researchers the opportunity that has integrated approach to understand the interaction of genetics, physiology and ecology.

We must emphasize that under certain conditions like rapid changes i.e. advent of war, earthquake, displacement of persons etc., the population tends to be impulsive in nature (see [2,8,16-18]). For this reason, we consider impulsive analog of the Von-Foerster-Makendrick model of an age-dependent population in given ecosystem. The model is typical impulsive partial differential equations and has potential applications in modelling the population of species stratified into age groups and epidemiology of infectious diseases like malaria and HIV/AIDs.

\section{B-Transform}

Consider the impulsive differential equations

$$
\begin{aligned}
& \dot{x}=f(t, x), t \neq t_{k}, k=0,1,2, \cdots \\
& \Delta x=I\left(x\left(t_{k}\right)\right)
\end{aligned}
$$

where

$$
\begin{aligned}
& f: R^{+} \times R^{n} \rightarrow R^{n} \\
& I: R^{n} \rightarrow R
\end{aligned}
$$

$f$ and $I$ are assumed to satisfy the continuity and Lipchitz conditions for the existence and uniqueness of solution of (IDE) (see [3,4]).

The B-transform of the function $x(t)$ with impulses at fixed moments $\left\{t_{k}\right\}, k=1,2, \cdots$ during the evolutionary process is $[1,11]$

$$
B_{n}^{1} x(t)=x_{c}(q)+x_{1}(q)
$$

where

$x_{C}(q)$ and $x_{\mathrm{I}}(q)$ are the $L_{c}$ and $L_{I}$ components of the $B$-transform and are defined as

$$
x_{c}(q)=L_{c} x(t)=\int_{0}^{\infty} \mathrm{e}^{-t / q^{n^{1}}} x(t) \mathrm{d} t, t \neq t_{k}, k=0,1,2, \cdots
$$

And

$$
x_{1}(q)=L_{1} x(t)=\sum_{t_{o}<t_{k}<t} \mathrm{e}^{-t_{k} / q^{n^{1}}} I\left(x\left(t_{k}\right)\right)
$$

where

$n^{\prime}=0,1,2, \cdots, n ; n^{\prime}$ is the order of the transform. For sake of simplicity, we will choose $n^{\prime}=1$. The advantage of taking $n^{\prime}=1$ lies in the derivation of the inverse transform.

The inverse transform for components of $x_{C}(q)$ and $x_{\mathrm{I}}(q)$ was obtained (see [1], Theorem 1) as follows:

$$
\begin{aligned}
& x_{c}(t)=\frac{1}{2 \pi i} \int_{v-i \infty}^{v+i \infty} x_{c}(q) \mathrm{e}^{s q} \mathrm{~d} q \\
& x_{1}(t)=\sum_{t_{o}<t_{k}<t} \psi\left(t_{k}, q\right) I\left(x\left(t_{k}\right)\right)
\end{aligned}
$$

The B-transform is valid in the union of the sets

$$
\begin{aligned}
& A=\left\{x(t) \in R^{n}: \int_{0}^{\infty} \mathrm{e}^{-t_{k} / q^{n^{\prime}}}|x(t)| \mathrm{d} t<m_{1},\right. \\
& \left.t \in R^{+}-\left\{t_{k}\right\}, k=1,2, \cdots, n^{\prime}=1,2, \cdots\right\}
\end{aligned}
$$

And

$$
\begin{aligned}
& B=\left\{I\left(x\left(t_{k}\right)\right) \in R^{n}: \sum_{t_{0}<t_{k}<t} \mathrm{e}^{-t_{k} / q^{n^{\prime}}}\left|I\left(x\left(t_{k}\right)\right)\right|<m_{2},\right. \\
& \left.n^{\prime}=1,2, \cdots\right\}
\end{aligned}
$$

And such that 


$$
\operatorname{Diam}(A \cup B)=\max \left(|x(t)|,\left|I\left(x\left(t_{k}\right)\right)\right|\right)<+\infty
$$

where |.| is the $n$-dimensional Euclidean norm, $m_{1}$ and $m_{2}$ are constants that are assumed to exit and finite.

\section{Impulsive Diffusion Problems}

Consider an example of application of B-transform to impulsive partial differential equations. Impulsive partial differential equations have so many interesting applications. We will consider a diffusion problem of a porous media of substrate whose concentration is given by $C(x, t)$ at time $t$ and a distance of $x$ from the source. This type of problem is very useful in drug administration. The underlying mathematical model can be stated as follows:

$$
\begin{gathered}
\frac{\partial C(t, x)}{\partial t}=-k_{i} C(t, x)+D_{i} \frac{\partial^{2} C(t, x)}{\partial x^{2}} \\
+k_{i} r_{i}(x(t-h))+\mu u_{i}(t), t \neq t_{k} \\
\Delta C_{i}\left(t=t_{k}, x\right)=I\left(C_{i}\left(t_{k}, x\right)\right), k=0,1,2, \cdots
\end{gathered}
$$

Subject to the boundary conditions

$$
\begin{aligned}
& \text { at } x=0, \frac{\partial C_{i}}{\partial x}(t, x)=0 \\
& \text { at } x=L, \frac{\partial C_{i}(t, x)}{\partial x}=0
\end{aligned}
$$

where

$C_{i}(t, x)$ is the concentration of the substance that diffuses across the apparent volume into gastrointestinal tracts at time $t$ and the thickness of the tract being $x$ units. $k_{i}^{\prime}$ and $k_{i}$ are some rate constants, $D_{i}$ the coefficients of diffusion.

$r_{i}(x(t-h))$ accounts for the biological gestation function lagged by a constant $C_{i}$.

$u_{i}(t)$ is the relevant biological control function that is responsible for osmo-regulation of the medium.

Using a transformation of variable, a kind of separation of variable per say, we can express $C_{i}(t, x)$ in the form $C(t, x)=C(t) C(x)$ where $C(t)$ and $C(x)$ are purely functions of $t$ and $x$ respectively. This separation works for this type of problem, for other forms of impulsive partial differential equations, it may not work. This kind of features makes the theory of impulsive differential equation to be expanding at a rapid rate of late, even though the discovery of new phenomena like "beat- ing effect", "loss of autonomy" and "dying effect" are hampering the rate of its development $([2,4,9,15])$.

Let us return to the problem, if we slot $C(t, x)$ into Equations (6) and (7), we get

$$
\begin{gathered}
\frac{\mathrm{d} C_{i}(t)}{\mathrm{d} t}+k^{\prime} C_{i}(t)=\alpha_{i}, t \neq t_{k}, k=0,1,2, \cdots \\
\frac{\mathrm{d}^{2} C_{i}(x)}{\mathrm{d}^{2} x}+\alpha_{i} C_{i}(x)=k_{i}^{\prime} r_{i}(x(t-h))+\mu_{i} u_{i}(t), \\
t \neq t_{k}, k=0,1,2, \cdots \\
\Delta C\left(t=t_{k}\right)=I_{i}\left(C\left(t_{k}\right)\right) \\
0<t_{0}<t_{1}<t_{2}<\cdots<t_{k} ; \\
\lim _{k \rightarrow \infty} t_{k}=+\infty, \alpha_{i}=\text { constants }, i=1,2, \cdots
\end{gathered}
$$

Subject to given boundary conditions applying the $B$-transform to Equations (10)-(12) and solving the equations we get

$$
\begin{aligned}
& C_{i}(q)=\left(k_{i}^{\prime}-\frac{1}{q}\right)^{-1} C_{0}-\frac{\alpha_{i} q^{2}}{k_{i}^{\prime} q^{-1}} \\
&+\sum_{t_{o}<t_{k}<t} \phi_{i}\left(t_{k}, q\right) I\left(C\left(t_{k}\right)\right) \\
& \phi_{i}\left(t_{k}, q\right)=\int_{c_{i}} \mathrm{~d} q \mathrm{e}\left(\frac{1}{t_{k}}-t_{q}\right)
\end{aligned}
$$

And solving Equation (11) we get

$$
\begin{aligned}
& C_{i}(x)= A_{i} \sin \sqrt{\alpha x}+\beta_{i} \cos \sqrt{\alpha x} \\
&+\int_{x_{O}}^{x} G_{i}(s, t)\left(r_{i}(x(s-h))+\mu_{i}(s)\right) \mathrm{d} s \\
& G_{i}(s, t)=\alpha_{i} \cos \left(\sqrt{\alpha_{i}}(t+s)\right) .
\end{aligned}
$$

Now, we determine the inverse of $C_{i}(q), i=1,2$ and the constants $A_{i}$ and $B_{i}$. Thus

$$
\begin{aligned}
C_{i}(t)= & \mathrm{e}^{k_{i} t} C_{o}+\frac{\alpha_{i}}{k_{i}^{2}} \mathrm{e}^{-t / k_{i}^{i}} \\
& +\sum_{t_{o}<t_{k}<t} \phi_{i}\left(t_{k}, t\right) I\left(C\left(t_{k}\right)\right), i=1,2, \cdots
\end{aligned}
$$

The values for constants $A_{i}$ and $B_{i}, i=1,2$ are found to be

$$
A_{i}=\frac{\alpha_{i} \cos \sqrt{\alpha t}\left(r_{i}(0)+\mu_{i} u_{i}(t)\right)}{\alpha_{i}}
$$


Therefore $C(t, x)$ given the require solution to the model, that is,

$$
\begin{aligned}
C(t, x) & =\left(\mathrm{e}^{-k_{i} t} C_{o}+\frac{\alpha_{i}}{k_{i}^{\prime}} \mathrm{e}^{-t / k_{i}}+\sum_{t_{o}<t_{k}<i} \phi_{t}\left(t_{k}, t\right) I\left(C\left(t_{k}\right)\right)\right) \\
& A_{i} \sin \sqrt{\alpha_{i}} x+\beta_{i} \cos \sqrt{\alpha_{i}} x+\int_{x_{o}} G_{i}(s, t) \\
& \left(r_{i}(x(t-h))+\mu_{i} u_{i}(t)\right) \mathrm{d} t \\
& =\left(\mathrm{e}^{-k_{i} t} C_{o}+\frac{\alpha_{i}}{k_{i}^{2}} \mathrm{e}^{-t / k}+\sum_{t_{o}<t_{k}<t} \phi_{i}\left(t_{k}, t\right)\right) \\
& \left(C_{i} \cos \left(\sqrt{\alpha_{i}} x+\beta_{i}\right)\right) \\
& +\int_{x_{o}} G_{i}(s, t)\left(r_{i}(x(t-h))+\mu u_{i}(t)\right) \mathrm{d} t
\end{aligned}
$$

where

$$
C_{i}=\sqrt{A_{i}^{2}+B_{i}^{2}}, \beta_{i}=\tan ^{-1}\left(\frac{A_{i}}{B_{i}}\right)
$$

The problem exhibits oscillatory behavior at

$$
x_{i}=\frac{(1+4 k) \pi-2 \beta_{i}}{2 \sqrt{\alpha_{i}}}
$$

If

$$
r_{i}(x(t-h))+\mu_{i} u_{i}(t)=0, i=1,2, \cdots
$$

At steady state, when $t \rightarrow \infty$;

$\lim _{t \rightarrow \infty} C(t, x)=C_{0}(x)$

where

$$
\begin{aligned}
& C_{s}(x)=\lim _{t \rightarrow \infty}\left(\sum_{t_{o}<t_{k}<t} \Phi_{i}\left(t_{k}, t\right) I\left(C\left(t_{k}\right)\right)\left(C_{i} \cos \left(\sqrt{\alpha_{i}} x+\beta_{i}\right)\right)\right. \\
& +\int_{x_{o}}^{x} G_{i}\left(s_{i} t\right)\left(r_{i}(x(s-h))+\mu_{i} u_{i}(s)\right) \mathrm{d} s .
\end{aligned}
$$

If the system does not oscillate about the points $x_{i}$ as enumerated above and if $C_{s}(x)=0$ it oscillates.

From the above derivation, drugs that are needed for prophylactic or chemotherapeutic purposes the concentration must not be allowed to oscillate about the equilibrium at the steady state. Drugs that are to be used for immunization should not oscillate about the equilibrium at steady state in order to have long residue effect in the blood.

\section{Impulsive Population Model}

We consider impulsive analog of the Von-Foerster-Makendrick model of an age-dependent population in given ecosystem. The equation is given by

$$
\begin{gathered}
\alpha_{1} \frac{\partial N}{\partial x}+\alpha_{2} \frac{\partial N}{\partial t}=-\mu(t, x, N) F(N(t, x)), t \neq t_{k} \\
N(x, 0)=\Phi(x) \\
N(0, t)=\int_{0}^{\infty} \gamma(x, t) N(x, t) \mathrm{d} t \\
N\left(x, t=t_{k}\right)=I\left(N\left(x, t_{k}\right)\right) \\
0<t_{0}<t_{1}<t_{2}<\cdots<t_{k}, \frac{\lim }{k \rightarrow \infty} t_{k}=+\infty .
\end{gathered}
$$

where $N: D \times R^{+} \rightarrow R, \quad \phi \in C^{\infty}\left(R^{+}\right), \quad R^{+}=[0,+\infty)$, $N(x, t)$ is the population of a single species in given ecological set-up at time $t$ and $x$ age group. If

$I\left(N\left(x, t_{k}\right)\right)>0$ substrate (biomass) is taken away and if $I\left(N\left(x, t_{k}\right)\right)<0$ biomass is added to the environment ([3]).

We must emphasize that under certain conditions like rapid changes i.e. advent of war, earthquake, displacement of persons etc., and the population tends to be impulsive in nature [18].

$$
\begin{gathered}
\mu: R^{+} \times D \times R^{+} \rightarrow R \\
F: R^{+} \rightarrow R^{+}
\end{gathered}
$$

$\Phi$ Is the growth rate, $F$ is a non-linear function and $\alpha_{i}, i=1,2$. are some relevant rate constants. Applying $B$-transform to model we arrive at

$$
\begin{aligned}
& L_{c} N(x, t)=N_{c}(x, q)=\int_{0}^{\infty} \mathrm{e}^{-t / q} N(x, t) \mathrm{d} t \\
& \begin{aligned}
L_{1} N(x, t)=N_{I}(x, q)=\sum_{t_{o}<t_{k}<t} \mathrm{e}^{q t_{k}} I\left(N\left(x, t_{k}\right)\right) \\
\begin{aligned}
L_{c}\left(\frac{\partial}{\partial x} N(x, t)\right) & =\int_{0}^{\infty} \frac{\partial}{\partial x} \mathrm{e}^{t / q} N(x, t) \mathrm{d} t=\frac{\partial}{\partial x} N(x, q) \\
& =\frac{\partial}{\partial x} \int_{0}^{\infty} \mathrm{e}^{t / q} N(x, t) \mathrm{d} t=\frac{\partial}{\partial x} N(x, q) \\
L_{c}\left(\frac{\partial}{\partial x} N(x, t)\right) & =\frac{\partial}{\partial x} \int_{0}^{\infty} \mathrm{e}^{t / q} N(x, t) \mathrm{d} t=\frac{1}{q} N_{c}(x, q) . \\
\alpha_{1} \frac{\partial}{\partial x} N_{c}(x, q) & +\frac{\alpha_{2}}{q} N_{c}(x, q)=\alpha_{2} \phi(x)-G(x, q) \\
G(x, q) & =-\int_{0}^{\infty} \mathrm{e}^{t / q} \mu(t, x, N) F(N(t, x)) \mathrm{d} t \\
N_{c}(0, q) & =-\int_{0}^{\infty} e^{t / q} \mathrm{~d} t \int_{0}^{\infty} \gamma\left(x, t_{1}\right) N\left(x, t_{1}\right) \mathrm{d} t \mathrm{~d} t_{1} .
\end{aligned} \\
=\int_{0}^{\infty} \int_{0}^{\infty} \mathrm{e}^{t / q} \gamma(x, t) N\left(x, t_{1}\right) \mathrm{d} t \mathrm{~d} t_{1} .
\end{aligned}
\end{aligned}
$$

Substituting the value of $N_{c}(x, q)$ and then solving Equation (26) for $N_{c}(x, q)$ for fixed $q$, we get 
$\frac{\mathrm{d} N_{c}(x, q)}{\mathrm{d} x}+\frac{a_{2}}{a_{1}} \frac{1}{q} N_{c}(x, q)=\frac{a_{2}}{a_{1}} \phi(x)-\frac{1}{a_{1}} G(x, q)$

$N_{c}(0, q)=\int_{0}^{\infty} \int_{0}^{\infty} \mathrm{e}^{-t / q} \gamma(x, t) N\left(x, t_{1}\right) \mathrm{d} t \mathrm{~d} t_{1}$.

Solving Equation (27) we get

$$
\begin{aligned}
& N_{c}(x, q)=N_{c}(0, q) \exp \left(-\int_{0}^{\infty} \frac{\alpha_{2}}{\alpha_{1}} \cdot \frac{1}{q} \mathrm{~d} s\right) \\
& N_{c}(x, q)=N_{c}(0, q) \exp \left(-\int_{0}^{\infty} \frac{\alpha_{2}}{\alpha_{1}} \cdot \frac{1}{q} \mathrm{~d} s\right)
\end{aligned}
$$

Therefore

$$
N(x, q)=N_{c}(x, q)+N_{1}(x, q) .
$$

It follows that

$$
\begin{aligned}
& N_{c}(x, t)=\frac{1}{2 \pi i} \int_{\mu-i \infty}^{\mu+i \infty} N_{c}(x, q) \mathrm{e}^{t q} \mathrm{~d} q \\
& N_{1}(x, q)=\sum_{t_{o}<t_{k}<t} \beta\left(t_{k}, t\right) I\left(N\left(x, t_{k}\right)\right)
\end{aligned}
$$

Let $C$ be contour for which the integration is carried out in the complex plain.

Therefore

$$
\begin{gathered}
N(x, t)=N_{c}(x, t)+N_{1}(x, t) . \\
N(x, t)=\frac{1}{2 \pi i}\left\{\int_{C} N_{c}(0, q) \exp \left(-\int_{a}^{x} \frac{a_{2}}{a_{1}} \frac{\mathrm{d} s}{q}+t q\right) \mathrm{d} q\right. \\
+\left[\int_{C} \exp \left(-\int_{a}^{x} \frac{a_{2}}{a_{1}} \frac{\mathrm{d} s}{q}+t q\right) \int_{a}^{x}\left(\frac{a_{2}}{a_{1}} \phi(s)-\frac{a_{2}}{a_{1}} G(x, q)\right)\right. \\
\left.\left.\exp \left(\int_{a}^{x} \frac{a_{2}}{a_{1}} \cdot \frac{1}{q} \mathrm{~d} s\right)\right] \mathrm{d} q \sum_{t_{0}<t_{k}<t} \mathrm{e}^{-t_{k} / q} I\left(N\left(x, t_{k}\right)\right)\right\}
\end{gathered}
$$

We note that the system has no solution passing through $\left(t^{*}, N\left(t^{*}\right)\right)$ if

$$
I\left(N_{i}\left(t, t_{k}\right)\right)=\frac{1}{C_{1} N\left(t, t_{k}\right)+\alpha_{2} t+C_{2}}, k=0,1,2, \cdots
$$

where $C_{i}$ are possitive constans such that

$$
t^{*}=t=\frac{-C}{\alpha_{2}} N\left(t^{*}, t_{k}\right)-\frac{C_{2}}{\alpha_{2}}, \alpha_{2}<0 .
$$

This model wills exhibits the 'dying effects', which mean that the solution is not continuous across some natural boundary containing the solution ([2-4]). To this particular model, this means that the population of the specie attains super saturation level $t^{*}$ such that after time $t>t^{*}$ the population starts showing some strange behavior that the population cannot be quantified.

The "dying effect" as $t>t^{*}$ suggests that the environment cannot accommodate the population of the spe- cie any more. By Darwin's theory a catastrophic stage is reached that only the fittest can survive beyond this regime (i.e. $t>t^{*}$ ); there expected to be sharp competition for food, shelter and waste disposal etc. It is interesting to note that dying effect does not exist if $\alpha_{2} \geq 0$.

We consider special cases of Equations (22)-(25).

Case I

When if $\mu=$ const and $F(N(x, t))=N(t, x)$.

Then

$$
N_{c}(x, q)=\frac{N_{c}(0, q) \psi(x)+\frac{\alpha_{2}}{\alpha_{1}} \psi(x) \int_{0}^{x} \phi(s) \mathrm{d} s}{1-\frac{\mu x}{\alpha} \psi^{2}(x)}
$$

$\Psi(x):=\exp -\frac{\alpha_{2}}{\alpha_{1}}\left(\frac{x}{q}\right)$. Thus using Equations (28), (30) \& (32), we find $N(x, t)$ for this particular case. If $\alpha_{2}<0, x>0, \mu>0$ and $x \ll \frac{\alpha_{2}}{\mu}$

Then

$$
N_{c}(q, x) \approx \frac{-\alpha_{2}}{\mu x \psi(x)}\left[N_{c}(0, q)+\frac{\alpha_{2}}{\alpha_{1}} \int_{0}^{x} \phi(s) \mathrm{d} s\right]
$$

By asymptotic expansion using Maple 15 Symbolic Programming at dos command prompt:

$>$ asympt $(\exp ($ theta $/ q), q)$;

$$
1+\frac{\theta}{q}+\frac{\theta^{2}}{2 q^{2}}+\frac{\theta^{3}}{6 q^{3}}+\frac{\theta^{4}}{24 q^{4}}+\frac{\theta^{5}}{120 q^{5}}+O\left(\frac{1}{q^{6}}\right)
$$

$>$ subs (theta $=$ alpha $[2] /$ alpha $[1]^{*} x, \%$ );

$$
\begin{aligned}
& 1+\frac{\alpha_{2} x}{\alpha_{1} q}+\frac{\alpha_{2}^{2} x^{2}}{2 \alpha_{1}{ }^{2} q^{2}}+\frac{\alpha^{3}{ }_{2} x^{3}}{6 \alpha_{1}{ }^{3} q^{3}}+\cdots+\frac{\alpha_{2}{ }^{5} x^{5}}{120 \alpha_{1}{ }^{5} q^{5}}+O\left(\frac{1}{q^{6}}\right) \\
& >\% * \exp \left(t^{*} q\right) ; \\
& \left(1+\frac{\alpha_{2} x}{\alpha_{1} q}+\frac{\alpha_{2}^{2} x^{2}}{2 \alpha_{1}{ }^{2} q^{2}}+\frac{\alpha_{2}^{3} x^{3}}{6 \alpha_{1}^{3} q^{3}}+\cdots+\frac{\alpha_{2}^{5} x^{5}}{120 \alpha_{1}^{5} q^{5}}+O\left(\frac{1}{q^{6}}\right)\right) \mathrm{e}^{t q}
\end{aligned}
$$

And the asymptotic expansion of $N_{I}\left(t_{k}, q\right)$ being

$$
\begin{aligned}
& N_{I}\left(t_{k}, q\right) \approx 6\left[I+O\left(\frac{1}{t^{6}}\right)\right] \mathrm{e}^{t q}+\sum_{k=0}^{5}\left\{\frac{\mathrm{e}^{t q}}{q} t_{k} I+\frac{\frac{1}{2} e^{t q} t_{k}^{2} I}{q^{2}}\right. \\
& \left.+\frac{\frac{1}{6} e^{t q} t_{k}^{3} I}{q^{3}}+\frac{\frac{1}{24} e^{t q} t_{k}^{4} I}{q^{4}}+\frac{\frac{1}{120} e^{t q} t_{k}^{5} I}{q^{5}}\right\}
\end{aligned}
$$

where the summation over is taken over

$$
I=I\left(N\left(t_{k}, t\right)\right), 0<t_{k}<t .
$$

Therefore 


$$
\begin{aligned}
& N(x, t) \\
& =\frac{1}{2 \pi i} \int_{C}\left(1+\frac{\alpha_{2} x}{\alpha_{1} q}+\frac{\alpha_{2}^{2} x^{2}}{2 \alpha_{1}{ }^{2} q^{2}}+\frac{\alpha_{2}^{3} x^{3}}{6 \alpha_{1}^{3} q^{3}}+\cdots+\frac{\alpha_{2}^{5} x^{5}}{120 \alpha_{1}^{5} q^{5}}\right) \\
& \mathrm{e}^{t p} N_{C}(0, q) \mathrm{d} q+O\left(\frac{1}{2 \pi i} \int_{C} \frac{N_{c}(0, q) \mathrm{e}^{t q} \mathrm{~d} q}{q^{6}}\right) \\
& +\frac{1}{4 \pi i} \int_{C}\left(\frac{\alpha_{2} \mathrm{e}^{t q}}{t} \int_{0}^{x} \phi(s) \mathrm{d} s \mathrm{~d} q\right)+\sum_{0<t_{k}<t} \beta\left(t_{k}, t\right) I\left(N\left(t_{k}\right)\right)
\end{aligned}
$$

Case II: For $\mu=$ const and
$F(N(t, N))=\frac{N}{K}(K-N), K$ is the saturation (maximum) population the environment can support. For this case, we found that

$$
\begin{aligned}
& \frac{\mu x}{K} \psi^{2}(x) N_{c}^{2}(x, q)+\left(1-\mu x \psi^{2}(x)\right) N_{c}(x, q) \\
& =N_{c}(0, q) \psi(x)+\frac{\alpha_{2}}{\alpha_{1}} \psi^{2}(x) \int_{\alpha}^{x} \phi(s) \mathrm{d} s,
\end{aligned}
$$

It follows that

$$
\begin{aligned}
& N_{c}^{+}(x, q) \\
& =\frac{1-\left(1-\mu x \psi^{2}(x)\right)+\sqrt{\left(1-\mu x \psi^{2}(x)\right)-\frac{4 \mu x}{K}\left(\psi^{3}(x) N_{c}(0, q)+\frac{\alpha_{2}}{\alpha_{1}} \psi^{2}(x) \int_{o}^{t} \phi(s) \mathrm{d} s\right)}}{\frac{2 \mu x}{K} \psi^{2}(x)} \\
& N_{c}^{-}(x, q) \\
& =\frac{1-\left(1-\mu x \psi^{2}(x)\right)-\sqrt{\left(1-\mu x \psi^{2}(x)\right)-\frac{4 \mu x}{K}\left(\psi^{3}(x) N_{c}(0, q)+\frac{\alpha_{2}}{\alpha_{1}} \psi^{2}(x) \int_{o}^{t} \phi(s) \mathrm{d} s\right)}}{\frac{2 \mu x}{K} \psi^{2}(x)}
\end{aligned}
$$

And $q_{0}$ is any real number.

If we slot Equations (33) and (34) into Equations (29) and (30) the solution to the model can be found for cases II and I respectively.

If we let

$$
\begin{aligned}
& A=\frac{\mu x}{K}, B=1+\mu \Psi^{2}(x), C=N_{c}(0, q) \Psi(x), \\
& D=\frac{\alpha_{2}}{\alpha_{2}} \Psi^{2}(x) \int_{\alpha}^{x} \phi(s) \mathrm{d} s \text { and } y=\frac{8 A^{2}}{B} .
\end{aligned}
$$

We can approximate $N_{c}^{ \pm}(x, q)$ in Taylors series using the Maple 15 software as

$$
\begin{aligned}
& N_{c}^{ \pm}(q, x) \approx \frac{B}{2 A}\left[1 \pm\left(1+\frac{1}{2} y-\frac{1}{8} y^{2}+\frac{1}{16} y^{3}\right.\right. \\
& \left.\left.-\frac{5}{128} y^{4}+\frac{7}{256} y^{5}+O\left(y^{6}\right)\right)\right]
\end{aligned}
$$

If $\mu \ll 1$ and $K$ is very large than

$$
N_{c}(q, x) \approx \frac{B}{2 A}\left[2+\frac{1}{2} y\right]
$$

Hence

$$
\begin{aligned}
& N_{c}(t, x) \\
& \approx \frac{K}{2 \pi \mu x} \int_{C}\left(1+\exp -2 \frac{\alpha_{2} x}{\alpha_{1} q}\right) \exp t q \mathrm{~d} q \rightarrow 0 \\
& \text { as } x \rightarrow+\infty
\end{aligned}
$$

Since $\int_{C} \exp t q \mathrm{~d} q$ is analytic in $C$ and by the Cauchy integration theorem it is zero.

The asymptotical expansion for $N_{c}(t, x)$ by Dos command prompt in Maple 15 we have:

$>N[c](x, q):=1 / 2 * \operatorname{pi} / i * \operatorname{int}(\% \% * N[c](0, q), q)+$ alpha $2 / 2 *$ $\operatorname{int}\left(\exp \left(t^{*} q\right), q\right) * \operatorname{int}(\operatorname{phi}(s), s=0 \ldots x)$;

$$
\begin{aligned}
& N_{c}(x, q)=\frac{K}{16 \mu x \pi^{2} i} \int_{C}\left(1+\frac{\alpha_{2} x}{\alpha_{1} q}+\frac{\alpha_{2}{ }^{2} x^{2}}{2 \alpha_{1}{ }^{2} q^{2}}+\frac{\alpha_{2}{ }^{3} x^{3}}{6 \alpha_{1}^{3} q^{3}}+\frac{\alpha_{2}{ }^{4} x^{4}}{24 \alpha_{1}{ }^{4} q^{4}}+\frac{\alpha_{2}{ }^{5} x^{5}}{120 \alpha_{1} q^{5}}\right) \\
& N_{c}(0, q) \mathrm{d} q+O\left(\frac{1}{4 \pi^{2}} \frac{K}{\mu x i} \int_{C} \frac{N_{C}(0, q) \mathrm{e}^{t q}}{q^{6}} \mathrm{~d} q\right)+\frac{1}{4} \frac{K}{\pi \mu x i}\left(\frac{\alpha_{2}}{t} \int_{C} \mathrm{e}^{t q} \mathrm{~d} q \int_{0}^{x} \phi(s) \mathrm{d} s\right)
\end{aligned}
$$

where the integration is take over a contour $\mathrm{C}$ containing the pole $q=0$ of order $k=6$. By the use of Cachy-
Goursat theorem, the integral can be reduced to only an integration around a small circle about the pole $q=0$ of 
order $k=6$. The value for $\int_{C} \frac{\mathrm{e}^{t q}}{q^{k}} \mathrm{~d} q$ can be obtained by the use of residue theorem as

$$
\begin{aligned}
& \int_{C} \frac{\mathrm{e}^{t q} \mathrm{~d} q}{q^{k}} \\
& =\left.2 \pi i \operatorname{Re} s\left(\mathrm{e}^{t q}\right)\right|_{q=0}=\left.2 \pi i \frac{1}{(k-1) !} \frac{\mathrm{d}^{(k-1)} \mathrm{e}^{t q}}{\mathrm{~d} x^{(k-1)}}\right|_{q=0} \\
& =2 \pi i \frac{t^{k}}{(k-1) !}
\end{aligned}
$$

Substitute the above value for $k=0,1,2, \cdots, 6$ gives the asymptotic approximation for $N_{C}(t, x)$ and $N(t, x)=N_{I}(t, x)+N_{C}(t, x)$ where

$$
\begin{aligned}
& N_{I}(t, x) \approx \sum_{0<t_{k}<t, k=0, \cdots, 5} \frac{1}{k !} t_{k}{ }^{k-1} t^{k} \\
& I\left(N\left(t, x\left(t_{k}\right)\right)+O\left(t^{6} I\left(N\left(t_{6}, x\left(t_{6}\right)\right)\right)\right)\right)
\end{aligned}
$$

By residue theorem which is unbounded as $t \rightarrow \infty$ and $x \rightarrow+\infty$ except if

$$
\left|I\left(N\left(t_{k}, x\left(t_{k}\right)\right)\right)\right| \leq \frac{k_{0}}{\left|x\left(t_{k}\right)\right|^{\delta}} \exp -\left(t-t_{k}\right) ;
$$

where $\delta \geq 1$ and $k_{0} \geq 0$ are constants.

The Maple was used to find $N_{c}(t, q)$ involving some special functions see the appendix and the use of the residue theorem can then be applied to complete the solution.

\section{Conclusions}

From the two models considered, the B-transform has been demonstrated to offer simple way for solving fixed moment impulsive system (see [19]) for more applications). More works need to be done on applications of B-transform especially taxism problems by studying the response of living organisms to stimulus. Moreover, in molecular chemistry, the use of impulsive diffusion model can be explored to investigate the movement of chemical substances in and out cells and tissues of organisms.

Furthermore, the Von-Foerster-Makendrick models can be further exploited to study epidemiology of infectious diseases like malaria and HIV/AIDS. In view of these, it is recommended that more work should be done on the applications of B-transform couple with the use symbolic programming approach to solve problems.

\section{Acknowledgements}

The authors are grateful to the National Mathematical Centre, Abuja, Nigeria and the Kaduna State University
Kaduna, Nigeria for their supports.

\section{REFERENCES}

[1] B. O. Oyelami and S. O. Ale, "B-transform Method and its Applications, in obtaining Solutions of some Impulsive Models," International Journal of Mathematical Education in Science and Technology, Vol. 31, No. 4, 2000, pp. 525-538. http://dx.doi.org/10.1080/002073900412633

[2] S. O. Ale and B. O. Oyelami, "Impulsive System and Applications," International Journal of Mathematical Education in Science and Technology, Vol. 31, No. 4, 2000, pp. 539-544. http://dx.doi.org/10.1080/002073900412642

[3] D. D. Bainov, V. Lakshikantham and P. S. Simeonov, "Theory of Impulsive Differential Equations," World Scientific Publication, Singapore, 1989.

[4] P. S. Simeonov and D. D. Bainov, "Theory of Impulsive Differential Equations: Periodic Solutions and Applications," Essex, Longman, 1993.

[5] E. Beltrami, "Mathematics for Dynamic Modeling," Academy Press, London, 1987.

[6] B. O. Oyelami, "On Military Model for Impulsive Reinforcement Functions using Exclusion and Marginalization Techniques," Nonlinear Analysis, Vol. 35, No. 8, 1999, pp. 947-958.

http://dx.doi.org/10.1016/S0362-546X(98)00114-X

[7] B. O. Oyelami, S. O. Ale, P. Onumanyi and J. A. Ogidi, "B-Transform Method and Application to Sickle Cell Aneamia," 2008, pp. 202-220.

http://sirius-c.ncat.edu/asn/ajp/allissue/ajp-ISOTPAND/in dex.html

[8] B. O. Oyelami and S. O. Ale, "On Existence of Solution, Oscillation and Non-Oscillation Properties of Delay Equations Containing 'Maximum,", Acta Applicandae Mathematicae Journal, Vol. 109, No. 3, 2010, pp. 683701. http://dx.doi.org/10.1007/s10440-008-9340-1

[9] P. S. Simeonov and D. D. Bainov, "Impulsive Differential Equation: Asymptotic Properties of the Solutions," World Scientific Publication, Singapore, 1989.

[10] S. G. Pandit and H. Deo Sudashiv, "Differential Systems Involving Impulsive. Lecture Notes in Maths," SpringerVerlag, Berlin-Heidelberg-New York, 1982.

[11] B. O. Oyelami and S. O. Ale, "B-Transform and Its Applications to a Fish-Hyacinth Model," International Journal of Mathematical Education in Science and Technology, Vol. 33, No. 4, 2002, pp. 565-573. http://dx.doi.org/10.1080/00207390210131353

[12] B. O. Oyelami, S. O. Ale, P. Onumanyi and J. A. Ogidi, "Impulsive HIV-1 Model in the Presence of Antiretroviral Drugs Using B-Transform Method," Proceedings of African Mathematical Union, Vol. 1, No. 1, 2003, pp. 6276.

[13] B. O. Oyelami, S. O. Ale and P. Onumanyi, "Impulsive HIV Model Using B-Transform," The Proceedings of $\mathrm{Na}$ tional Mathematical Centre on Conference on Computational Mathematics, Vol. 2, No. 1, 2005, pp. 50-64. http://nmcabuja.org/nmc_proceedings.html 
[14] B. Davies, "Brian Davies Integral Transforms and Their Applications," Springer Publisher, Berlin-HeidelbergNew York, 2002.

[15] M. B. V. Robert, "Biology; a Functional Approval," Nelson Butler and Tanner Ltd., Rome and London, 1971.

[16] V. Lakshimikantham and Z. Dric, "Positivity and Boundedness of Solutions of Impulsive Reaction-Diffusion Equations," Journal of Computational and Applied Mathematics, Vol. 88, No. 1, 1988, pp. 175-184. http://dx.doi.org/10.1016/S0377-0427(97)00210-0

[17] L. H. Erbe, H. I. Freeman, X. Z. Lin and H. J. Wu, "Com- parison Principles for Impulsive Parabolic Species Growth," The Journal of the Australian Mathematical Society, Series B, Vol. 32, No. 4, 1991, pp. 382-400.

[18] S. O. Ale and B. O. Oyelami, "On Chemotherapy of Impulsive Models Involving Malignant Cancer Cells," Abacus, Journal of Mathematical Association of Nigeria, Vol. 24, No. 2, 1996, pp. 1-10.

[19] B. O. Oyelami and S. O. Ale, "Impulsive Differential Equations and Applications to Some Models: Theory and Applications. A Monograph," Lambert Academic Publisher, Saarbrücken, 2012. 


\section{Appendix}

$>$ approx $2:=\operatorname{asympt}(1+\exp ($ theta/q),$q)$;

approx $2:=2+\frac{\theta}{q}+\frac{\theta^{2}}{2 q^{2}}+\frac{\theta^{3}}{6 q^{3}}+\frac{\theta^{4}}{24 q^{4}}+\frac{\theta^{5}}{120 q^{5}}+O\left(\frac{1}{q^{6}}\right)$

$>$ Subs (theta $=-2 *$ alpha $2 * x /$ alpha $1, \%)$;

$$
\begin{aligned}
& 2-\frac{2 \alpha 2 x}{\alpha 1 q}+\frac{2 \alpha 2^{2} x^{2}}{\alpha 1^{2} q^{2}}-\frac{4 \alpha 2^{3} x^{3}}{3 \alpha 1^{3} q^{3}} \\
& +\frac{2 \alpha 2^{4} x^{4}}{3 \alpha 1^{4} q^{4}}-\frac{4 \alpha 2^{5} x^{5}}{15 \alpha 1^{5} q^{5}}+O\left(\frac{1}{q^{6}}\right) \\
& N(t, x):=\left(\left(\mathrm{e}^{(t q)^{2}}\right)+\frac{2 \alpha 2 x t E i(1,-2 t q)}{\alpha 1}+\frac{2 \alpha 2^{2} x^{2} t^{2}\left(-\frac{\left(\mathrm{e}^{(t q)^{2}}\right)}{t q}-2 E i(1,-2 t q)\right.}{\alpha 1^{2}}\right) \\
& -\frac{4}{3} \frac{\alpha 2^{3} x^{3} t^{3}\left(-\frac{\left(\mathrm{e}^{(t q)^{2}}\right)}{t^{2} q^{2}}-\frac{\left(\mathrm{e}^{(t q)^{2}}\right)}{t q}-E i(1,-2 t q)\right)}{\alpha 1^{3}} \cdots+\frac{2 \alpha 2^{2} x^{2} t^{2}\left(-\frac{\left(\mathrm{e}^{(t q)^{2}}\right)}{t q}-2 E i(1,-2 t q)\right)}{\alpha 1} \\
& -\frac{4}{15} \frac{\alpha 2^{5} x^{5} t^{5}\left(-\frac{1}{4} \frac{\left(\mathrm{e}^{(t q)^{2}}\right)}{t^{4} q^{4}}-\frac{1}{6} \frac{\left(\mathrm{e}^{(t q)^{2}}\right)}{t^{3} q^{3}}-\frac{1}{3} \frac{\left(\mathrm{e}^{(t q)^{2}}\right)}{t q}-\frac{2}{3} E i(1,-2 t q)\right) t}{\alpha 1^{5}} \\
& O\left(-\frac{1}{5} \frac{\left(\mathrm{e}^{(t q)^{2}}\right)}{t^{5} q^{5}}-\frac{1}{10} \frac{\left(\mathrm{e}^{(t q)^{2}}\right)}{t^{4} q^{4}}-\frac{1}{15} \frac{\left(\mathrm{e}^{(t q)^{2}}\right)}{t^{3} q^{3}}-\frac{1}{15} \frac{\left(\mathrm{e}^{(t q)^{2}}\right)}{t^{2} q^{2}}-\frac{2}{15} \frac{1}{15} \frac{\left(\mathrm{e}^{(t t)^{2}}\right)}{t q}-\frac{4}{15} E i(1,-2 t q)\right) \\
& \frac{2}{3} \frac{\alpha 2^{4} x^{4} t^{4}\left(-\frac{1}{3} \frac{\left(\mathrm{e}^{(t q)^{2}}\right)}{t^{3} q^{3}}-\frac{1}{3} \frac{\left(\mathrm{e}^{(t q)^{2}}\right)}{t^{2} q^{2}}-\frac{2}{3} \frac{\left(\mathrm{e}^{(t q)^{2}}\right)}{t q}-\frac{4}{3} E i(1,-2 t q)\right.}{\alpha 1^{4}}\left[-\frac{4}{15} \operatorname{Ei}(1,-2 t q)\right] w \\
& =\frac{1}{2}\left[\mathrm{e}^{(t q)^{2}}+\frac{2 \alpha 2 x t E i(1,-2 t q)}{\alpha 1}\right]+\frac{2 \alpha 2^{2} x^{2} t^{2}\left(-\frac{\left(\mathrm{e}^{(t q)^{2}}\right)-2 E i(1,-2 t q)}{t q}\right)}{\alpha 1^{2}} \\
& -\frac{4}{15} \operatorname{Ei}(1,-2 t q) K /(\pi \mu x) \\
& w=\frac{K}{2 \pi \mu x}
\end{aligned}
$$

$>$ Subs (alpha1 $=0.001$, alpha2 $=0.05, \mathrm{pi}=3.142, K=1000, \%)$;
$>\% * \exp \left(t^{*} q\right)$

$$
\begin{aligned}
& \left(2-\frac{2 \alpha 2 x}{\alpha 1 q}+\frac{2 \alpha 2^{2} x^{2}}{\alpha 1^{2} q^{2}}-\frac{4 \alpha 2^{3} x^{3}}{3 \alpha 1^{3} q^{3}}\right. \\
& \left.+\frac{2 \alpha 2^{4} x^{4}}{3 \alpha 1^{4} q^{4}}-\frac{4 \alpha 2^{5} x^{5}}{15 \alpha 1^{5} q^{5}}+O\left(\frac{1}{q^{6}}\right)\right) \mathrm{e}^{(t q)}
\end{aligned}
$$




$$
\left.\begin{array}{c}
\mathrm{e}^{(t q)^{2}}+100 \cdot x t E i(1,-2 t q)+5000 \cdot x^{2} t^{2}\left(-\frac{\left(\mathrm{e}^{(t q)^{2}}\right)}{t q}-2 E i(1,-2 t q)\right) \\
-166666.6666 x^{3} t^{3}\left(-\frac{1}{2} \frac{\left(\mathrm{e}^{(t q)^{2}}\right)}{t^{2} q^{2}}-\frac{\left(\mathrm{e}^{(t q)^{2}}\right)}{t q}-2 E i(1,-2 t q)\right)+\cdots \\
+0.4166666667 \cdot 10^{7} \cdot x^{4} t^{4}\left(-\frac{1}{3} \frac{\left(\mathrm{e}^{(t q)^{2}}\right)}{t^{3} q^{3}}-\frac{1}{3} \frac{\left(\mathrm{e}^{(t q)^{2}}\right)}{t^{2} q^{2}}-\frac{2}{3} \frac{\left(\mathrm{e}^{(t q)^{2}}\right)}{t q}-\frac{4}{3} E i(1,-2 t q)\right) . \\
{\left[-\frac{1}{5} \frac{\left(\mathrm{e}^{(t q)^{2}}\right)}{t^{5} q^{5}}-\frac{1}{10} \frac{\left(\mathrm{e}^{(t q)^{2}}\right)}{t^{4} q^{4}}-\frac{1}{15} \frac{\left(\mathrm{e}^{(t q)^{2}}\right)}{t^{3} q^{3}}-\frac{1}{15} \frac{\left(\mathrm{e}^{(t q)^{2}}\right)}{t^{2} q^{2}}-\frac{2}{15} \frac{\left(\mathrm{e}^{(t q)^{2}}\right)}{t q}-\frac{4}{15} E i(1,-2 t q)\right] /(\mu x)}
\end{array}\right] / .
$$

